\title{
Acknowledgement to Reviewers of Symmetry in 2016
}

\author{
Symmetry Editorial Office
}

Published: 26 January 2017

MDPI AG, St. Alban-Anlage 66, 4052 Basel, Switzerland; symmetry@mdpi.com

The editors of Symmetry would like to express their sincere gratitude to the following reviewers for assessing manuscripts in 2016.

We greatly appreciate the contribution of expert reviewers, which is crucial to the journal's editorial process. We aim to recognize reviewer contributions through several mechanisms, of which the annual publication of reviewer names is one. Reviewers receive a voucher entitling them to a discount on their next MDPI publication and can download a certificate of recognition directly from our submission system. Additionally, reviewers can sign up to the service Publons (https://publons.com) to receive recognition. Of course, in these intiatives we are careful not to compromise reviewer confidentiality. Many reviewers see their work as a voluntary and often unseen part of their role as researchers. We are grateful to the time reviewers donate to our journals and the contribution they make.

If you are interested in becoming a reviewer for Symmetry, see the link at the bottom of the webpage http://www.mdpi.com/reviewers.

The following reviewed for Symmetry in 2016:

Abay-Asmerom, Ghidewon

Aceves, Alejandro B.

Al Ameen, Moshaddique

Alberto, Pedro

Alcantud, José Carlos R.

Al-Dmour, Hayat

Ali, S. Twareque

Alkofer, Reinhard

Al-Masri, Eyhab

Alonso, Daniel

Alvarez Rodríguez, José María

Álvarez-Arenas, Tomás Gómez

Alvaro, Francisco

Amatya, Anup

Anagnostou, Dimitris E.

Anco, Stephen

Antonopoulos, Angelos

Antucheviciene, Jurgita

Arjunan, Sridhar Poosapadi

Arminjon, Mayeul

Arrigo, Daniel J.

Astrain Escola, José Javier
Attya, Mohamed

Awanou, Gerard

Azzopardi, George

Bäckström, Tom

Bakaoukas, Anastasios G.

Bamba, Kazuharu

Bamba, Kazuharu

Barbosa, Ramiro S.

Barghout, Lauren

Bates, Larry

Bauer, Corinna

Beer, Martin

Belmonte-Beitia, Juan

Benítez López, Julio

Berchtold, André

Bernabei, Rita

Bernabéu Alberola, José

Bernardin, Cédric

Bhuiyan, Mansurul

Bila, Nicoleta

Bohr, Jakob

Bojic, Iva
Bonisoli Alquati, Andrea

Bonyadi, Mohammad Reza

Borodich, Feodor M.

Bosisio, Ada Vittoria

Bouganis, Christos-Savvas

Bouma, Henri

Bradshaw-Hajek, B.H.

Brambilla, Nora

Brandt, Steven R.

Brevik, Iver Håkon

Broadbridge, Philip

Browne, Philip

Brunie, Nicolas

Buckeridge, John

Budker, Dmitry

Burke, Darren

Burrascano, Pietro

Bustince, Humberto

Caboussat, Alexandre

Caldelli, Roberto

Callico, Gustavo M.

Calzetta, Esteban 
Camacho, José

Campanelli, Leonardo

Campoamor-Stursberg, R.

Capozziello, Salvatore

Capozziello, Salvatore

Carbon, Claus-Christian

Castanon, David

Castillo, Kenier

Castle, Toen

Casula, Michele

Cavoretto, Roberto

Chae, Gyusoo

Chan, Yu-Wei

Chang, Elizabeth

Chang, Hsie-Chia

Charalampos, Pitas

Chen, Chuan-Hung

Chen, Elizabeth R.

Chen, Long

Cheng, Chuen-Sheng

Chern, C.S.

Chew, Lockyue

Chi, Hongmei

Chitsaz, Hamidreza

Choi, Sangil

Choo, Raymond

Chowdhury, Morshed

Chung, Yao-Liang

Claessens, Leon

Claude, Julien

Cohen, Thomas

Coniglio, A.

Constantinou, Anthony

Contini, Alessandro

Cortes, Jose

Costanzo, Sandra

Cotanch, Stephen R.

Couchot, Jean-François

Crutcher, Sihon H.

Cuevas-Maraver, Jesús

Cui, Zhenlu

Dale, Roberto

Dalton, Lori
Damianov, Damian

Danca, Marius-F.

David, Sergio

Davidsson, Paul

De Adana, Francisco Saez

De Bie, Hendrik

Dehmer, Matthias

Dehmer, Matthias

Deléaval, Luc

DeLellis, Pietro

Delis, Alex

Demni, Nizar

Desai, Alok

DeSesso, John M.

Deutsch, Emeric

Di, Jia

Di Crescenzo, Antonio

Dias, Jerry

Dimitriadis, Yannis

Dinges, Laslo

Dodgson, Neil

Dolgushev, Maxim

Donelli, Massimo

Donzelli, Sabrina

Döring, Maik

Dorst, Leo

Dou, Dejing

Droghei, Riccardo

Egger, Jan

El Shahat, Adel

Elena, Lucchi

Enokido, Tomoya

Espinasa, Luis

Estrada-Moreno, Alejandro

Fang, Shih-Hau

Fariborz, Amir

Fearnhead, Paul

Fernandez, Michael

Fernandez Fernandez, Luis ManueHale, Jack

Fino, Anna

Fischer, Ingrid

Fischer, Jean-Paul

Fisher, Mark
Florens, Serge

Fotiadis, Dimitrios

Fowler, Patrick

Freeman, D. Carl

Freeman, Eric

Freire, David

Fukuta, Naoki

Fulginei, Francesco Riganti

Gabbar, Hossam

Gadouleau, Maximilien

Gallicchio, Claudio

Gambi, Ennio

Gan, Guojun

Gandarias, Maria

Garcia, Andres Carrion

Gawhary, O. El

Gazeau, Jean Pierre

Genest, Vincent X.

Gentil, Christian

Giribet, Gaston

Giunti, C.

Glykas, Michael Markos

Gómez-Déniz, Emilio

Gonçalo Oliveira, Hugo

González-Díaz, Humberto

Gopalakrishnan, Bhaskaran

Gope, Prosanta

Goswami, Prashant

Goto, Yasuhiro

Gough, John

Graefe, Eva-Maria

Graham, Daniel

Green, Ben J.

Grøn, Øyvind

Groote, Stefan

Gros, Claudius

Guguchia, Zura

Hakansson, Anne

Hallgrimsson, Benedikt

Han, Daehee

Han, Jin-woo

Han, Jungong 
Han, Kwan Hee

Hayashi, Takashi

Hehl, Friedrich W.

Henkel, M.

Herbert, Andrew M.

Hereman, Willy A.

Hernández-Gómez, Juan Carlos

Herrera-Viedma, Enrique

Hidalgo, Arturo

Hintz, Andrew

Hochenegger, Andreas

Holvad, Torben

Holzinger, Andreas

Holzinger, Andreas

Hoogeveen, Ellen K.

Hornak, Larry A.

Hosaka, Atsushi

Hsia, Chih-Hsien

$\mathrm{Hu}$, Rui

$\mathrm{Hu}$, Shi-Min

$\mathrm{Hu}$, Yong

Huang, Fenix

Huang, Tony

Humphries, Stephen

Hussain, Zahir M.

Ibragimov, Nail

Ieong, Ricci

Ihara, Y.

Iliev, Plamen

Ishaq, Isam

James, Simon

Jang, Byungchul (Brandon)

Jang, Gun Ho

Jeong, Seon-phil

Jepps, Owen G.

Jiang, Hao

Jiang, Jie-Hong Roland

Jiang, Yufei

Jin, Yan

Joglekar, Yogesh

Joshi, Chaitanya

Jossen, Andreas

Jung, Juyoung
Kafatos, Menas

Kaimakamis, George

Kaiser, Norbert

Kajanus, Miika

Kalantari, Bahman

Kang, Dun-Yen

Kang, Jian

Kannan, Ravi

Kapadia, Mubbasir

Karimi, Hamid Reza

Karypis, George

Kashino, Kunio

Katsurada, Masanori

Kavic, Michael

Keedwell, Ed

Keller, Julien

Kelly, Gabrielle

Kersting, Kristian

Kerzel, Dirk

Khalil, M. Sayim

Khouider, Boualem

Kiefer, Ann

Kim, Bom Soo

Kim, Chang-Hun

Kim, Donghoon

Kim, Duck Young

Kim, Kyeong-seop

Kim, Taekyun

Kirillov, Oleg N.

Knappe, Svenja

Kohler, Sigmund

Kok, Wouter

Kolasiński, Piotr

Kong, Jun

Kopf, Stephan

Kordelas, Georgios A.

Kostelecky, Alan

Kotropoulos, Constantine

Kotsopoulos, Sotirios D

Kravari, Kalliopi

Kreider, Kevin L

Kreinovich, Vladik

Krüger, Björn
Kunkel, Julian

Lacoste, David

Laederach, Alain

Laefer, Debra

Lai, Hollis

Lane, Charles D.

Langbein, Frank C.

Lau, Rynson W. H.

Law, Rob

Le Moan, Steven

Lee, Gyu Myoung

Lee, Gyu Myoung

Lee, Jae Won

Lee, Jae Woong

Lee, Jeong-Yup

Lee, Kang-Hoon

Lee, Sanghyun

Lee, Sharon

Lee, Sze Sing

Lee, Taikjin

Leopold, Undine

Lewkowicz, Meir

Li, Gang

Li, Rao

Liang, Haozhao

Liao, Shaolin

LIM, Gilbert

Lin, Chin-Feng

Lin, Daw-tung

Lin, Jeng-wen

Liu, Bing

Liu, Xueting

Liu, Yanxi

Lombardi, Flavio

LoSecco, John M.

Lozano, Antoni

Lucas, J. De

Lucas, Pascual

Luo, xiaowei

Lyu, Siwei

Ma, Wenxiu

Malek, Mina Abdel

Malomed, Boris 


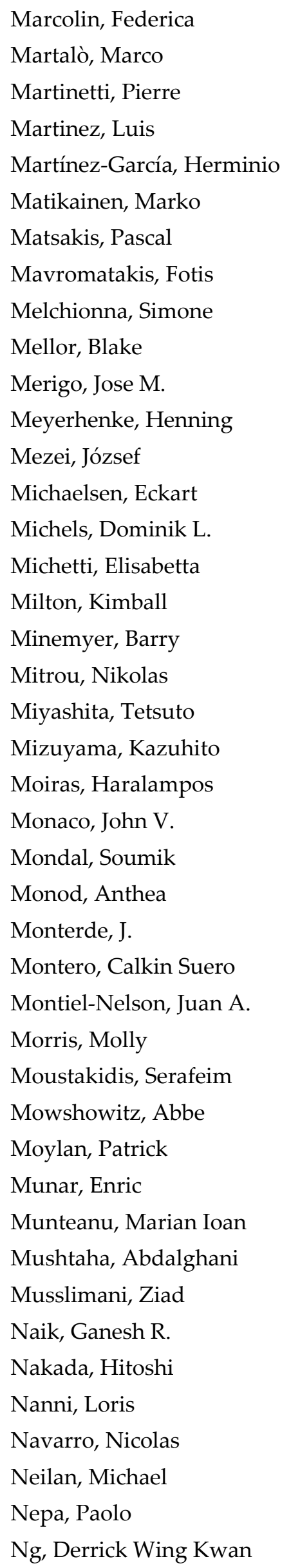

Ngatchou-Wandji, Joseph

Nieto, Juan J.

Ning, Wei

Nissen, Robert M.

Nitta, Muneto

Nowak, Piotr

Nucci, Maria Clara

Nyang, DaeHun

O'Connell, Robert F.

Oikonomou, V.K.

Oikonomou, Vasilis K.

Olaizola, Igor García

Oliveri, Francesco

O'Shaughnessy, Douglas

Palafox, Leon

Palasantzas, George A.

Panagiotakis, Costas

Papakostas, George A.

Pappas, Janice

Parastar, Amir

Park, Chansik

Park, Jaehyun

Park, Sanghoon

Paschos, E. A.

Patrizia, Boracchi

Peleg, Smadar

Pelinovsky, Dmitry

Peng, Linyu

Peng, $X i$

Penne, Rudi

Perea, Manuel

Peronnet, Frederique

Pertoldi, Cino

Peterson, Douglas

Petri, Giovanni

Pezanis-Christou, Paul

Pham, Thanh-Trung

Phillips, Anthony

Piraveenan, Mahendrarajah

Pliukhin, Oleksii

Polak, Michal

Polak, Michal

Pollock, Sara
Polychroniou, Panagiotis

Poplawski, Nikodem

Pouke, Matti

Profumo, Stefano

Pyron, Mark

Qin, Zhengrui

Qing, Xianming

Qu, Guangzhi

Quarati, Piero

Randles, Amanda

Rebel, Brian

Reed, Kyle

Reed, Matthew P

Reynaud, Roger

Riera, Juan Vicente

Rodríguez, Dionisio

Rodríguez, José M.

Roland, Michael

Rosensteel, George

Rossi, Luca

Roy, Swapnoneel

Rumí, Rafael

Russell, Neil

Rutle, Adrian

Saadatmand, Mehrdad

Sadun, Lorenzo A.

Sainz, Nekane Ione

Sampalli, Srinivas

San-Segundo, Rubén

Santoso, Fendy

Saridakis, Emmanuel

SATO, Hiroyuki

Satorra, Albert

Sauveron, Damien

Savriama, Yoland

Sawada, Tadamasa

Scala, Antonio

Scanlon, Mark

Scardicchio, Antonello

Schein, Stan

Schlager, Stefan

Schniederjans, Dara

Schuman, Catherine D. 
Scioscia, Floriano

See, Chan H.

Sepasi, Saeed

Sfyris, Dimitris

Shan, Gongbing

Shan, Xuechuan

Sharp, Philip

Shcherbakov, Victor

Shen, Jun

Sheng, Qin

Shieh, Hsin-Jang

Shikano, Takahito

Shinoda, Masato

Sigl, Günter

Sivaraman, Vaidy

Skilton, Mark

Slater, Paul B

Sohn, Bong-Soo

Spanò, Dario

Stecker, Floyd W.

Stentoumis, Christos

Stiebig, Helmut

Stokman, Jasper

Stork, André

Su, Yi-Sheng

Sun-Yuan, Hsieh

Susanto, Hadi

Svetovoy, Vitaly B.

Takahashi, Kazuo H.

Takemoto, Kazuhiro

Takenori, Obo

Tams, Benjamin

Tardif, Pierre Martin

Taronna, Massimo

Tasson, Jay

Teng, Shyh Wei

Teodorescu, Razvan

Tiakas, Eleftherios

Tian, Feng

Tiddeman, Bernie

Török, Marianna

Torrens, Francisco

Torres-Sospedra, Joaquin
Torrisi, Mariano

Tovar, Andres

Tracina, Rita

Tramontana, Fabio

Tranquada, John M.

Tron, Roberto

Trovati, Marcello

Trunfio, Giuseppe A.

Tsangaratos, Paraskevas

Tsou, Nien-Ti

Tutumluer, Erol

Ueckerdt, Torsten

Ujang, Uznir

Vafi, Sina

Vaknin, David

Valveny, Ernest

Van Der Meer, Th.H.

Van Dongen, Stefan

Van Dongen, Stefan

Vardanega, Tullio

Verma, Sanny

Vezzetti, Enrico

Vignat, Christophe

Vitali, David

Wagner, Stefan Rahr

Waldron, Andrew

Walters, E. George

Wang, Fu-Kwun

Wang, Gai-Ge

Wang, Ming-shi

Wang, Pei Jen

Wang, Shaohui

Wang, Yao-tien

Wang, Yongqi

Wang, Yumeng

Wang, Yun

Wazwaz, Abdul-Majid

Wei, Hua-Liang

Weitzen, Jay A.

West, Peter

Westhoff-Bleck, Mechthild

Whitlock, Michael

Wierling, August
Wilder, John

Winternitz, Pavel

Won Yoon, Dae

Woodruff, David

$\mathrm{Wu}$, Tzong-Chen

$\mathrm{Wu}$, Wentao

Xiang, Weidong

Yagi, Kent

Yang, Bo

Yang, Guang

Yang, Hyunseok

Yazdani, Morteza

Yelin, Susanne

Yen, Neil

Yoon, Seung-Hyun

You, Shaodi

You, Shingchern

You, Tae-soo

Young, Paul T.

$\mathrm{Yu}$, Xinghuo

Yuan, Zhongyi

Yun, Unil

Zarpelão, Bruno

Zavadskas, Edmundas Kazimieras

Zehmakan, A. Noori

Zervakis, Michalis

Zezyulin, Dmitry A.

Zhang, Bowu

Zhang, Dong

Zhang, Lei

Zhang, Longhe

Zhao, Qiangfu

Zhao, Shengkui

Zheng, Yalin

Zhou, Huiyu

Zhou, Xiran

Zhu, Hongmei

Zhuang, Hanqi

Zimmerman, William

Zou, Xukai

Zuber, Jean-Bernard 
(C) 2017 by the authors; licensee MDPI, Basel, Switzerland. This article is an open access article distributed under the terms and conditions of the Creative Commons Attribution (CC-BY) license (http://creativecommons.org/licenses/by/4.0/). 